\title{
The role of the source versus the collisionality in predicting a reactor density profile as observed on ASDEX Upgrade discharges
}

\author{
E. Fable, C. Angioni, V. Bobkov, J. Stober, R. Bilato, G. D. \\ Conway, T. Goerler, R. M. McDermott, T. Puetterich, M. \\ Siccinio $^{1}$, W. Suttrop, M. Teschke, H. Zohm, and the ASDEX \\ Upgrade Team \\ Max-Planck-Institut für Plasmaphysik, 85748 Garching, Germany \\ 1 also at EUROfusion \\ E-mail: emiliano.fable@ipp.mpg.de
}

\begin{abstract}
The design and optimization of a future tokamak fusion reactor, from the point of view of the plasma performance and output fusion power, requires the understanding of the underlying physics and the validation on present experiments of the theory-based tools used for the predictions. Present experimental efforts are devoted to approach reactor-relevant parameters (collisionality, $\beta$, normalized heat fluxes and sources, temperatures ratio) as much as possible.

In this work a series of discharges performed on ASDEX Upgrade are presented, where plasma density and auxiliary power levels are scanned to cover a parameter space that moves towards reactor relevant parameters. On this dedicated discharge dataset, a first-principle-based model is then applied for validation. The degree of agreement between code results and experimental measurements is shown and discussed. In cases where discrepancy is found, possible causes are identified.

It is shown that the employed modeling tool can predict the overall trend, consistent with more fundamental theoretical considerations, although quantitative extrapolation still has to be performed with care. Key results of this work show that the density peaking is mainly sustained by turbulence, with a minor role for the fueling source, and how this experimental demonstration is important to predict future reactor density profiles. Moreover, the role of electromagnetic effects is also pointed out.
\end{abstract}




\section{Introduction}

Demonstration of the viability of a fusion reactor for electricity production is of the highest priority and a fundamental stepping-stone in the European Roadmap to fusion [1]. In this framework, the design of prototypes for a pilot fusion reactor power plant based on the tokamak concept requires predictive tools to assess the performance of the core plasma and the resulting fusion power as a function of engineering parameters. This coupling between plasma physics and engineering is a key ingredient to successfully predict how an actual reactor would fare in a commercial sense $[2,3,4]$.

Even before going into the complex coupling between plasma and technological issues, one faces the problem of predicting the core plasma parameters given the machine size/field/current, available auxiliary power, and desired plasma density. Once the engineering parameters are defined, one could then convert them into dimensionless parameters such as $\beta$ ( $\beta$ being the ratio of plasma to magnetic pressures), collisionality, safety factor, normalized heat, particle and momentum fluxes (with respect to gyroBohm scaling), and defined ratios of electron to ion temperatures and heat fluxes. These parameters would then be used to perform experiments in present machines, trying to match those values, as much as possible simultaneously.

For the maximization of the fusion power in a DT-based reactor, one can look at the three elements that appear in the fusion power formula: the fuel density $n_{\mathrm{D}}, n_{\mathrm{T}}$, and the ion temperature $T_{\mathrm{i}}$. One can conveniently recast the fuel dependence in terms fuel mix $n_{\mathrm{D}} /\left(n_{\mathrm{D}}+n_{\mathrm{T}}\right)$, electron density $n_{\mathrm{e}}$, and impurity dilution, where $n_{\mathrm{e}}$ would be the observable/control parameter. The exponent of $n_{\mathrm{e}}$ in the fusion power expression is always 2 , while for $T_{\mathrm{i}}$, it depends on the temperature itself.

To justify putting in evidence the electron density, there are several reasons. For once, in present machines the electron density is preferentially measured for reasons of technical simplicity and accuracy. As such, modeling the electron density makes more sense to compare with present experiments. Second, if one assumes low ash content, which is a stringent requirement for a commercially viable reactor, then by quasi-neutrality fuel density will closely follow the electron density. Since the latter is dominantly driven by turbulence processes as shown later, one does not need to go into the details of ion transport.

At "low" core temperatures (e.g. $\sim 15-20 \mathrm{keV}$ ) the fusion cross-section depends roughly on $T_{\mathrm{i}}^{2}$, while at higher temperatures the exponent decreases towards 0 . This is depicted in figure 1. As such, this offers a particularly strong reason to focus on the behavior of the electron density profile. In figure 1, the behavior of quantities related to the fusion reactivity are displayed as a function of the plasma temperature. It is clear that for the high-temperature plasmas $\left(T_{\mathrm{i}}>30 \mathrm{keV}\right)$ foreseen in machines larger than ITER, e.g. DEMO $[5,6,7]$, one expects a much stronger impact of the density profile than that of the ion temperature profile on the obtained fusion power. As such, predicting the electron density peaking is of fundamental importance. 


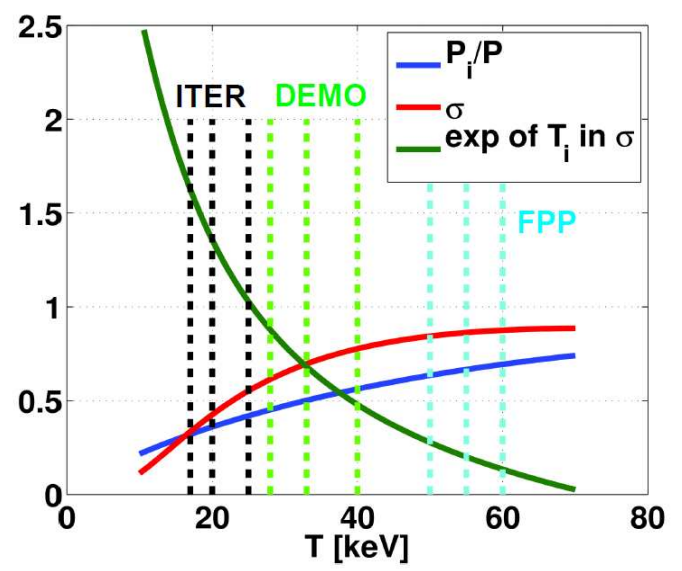

Figure 1. Dependence of the ratio of alpha power to ions $P_{\alpha, \mathrm{i}} / P_{\alpha}$, fusion cross-section $\sigma$, and the exponent of $T_{\mathrm{i}}$ in the cross-section, as a function of the central ion temperature. Typical values for three machines (ITER, DEMO, and an even larger fusion power plant FPP) are also displayed.

A critical assessment regarding this profile is the relative role of the fueling source and the intrinsic transport mechanisms (i.e. turbulence and neoclassica-driven particle convection), and how to extrapolate this relationship in a meaningful way to a reactor scenario. In the past there have been work that stressed the importance of the fueling source in $\mathrm{H}$-mode scenarios on JET $[8,9]$. This has also been recently pointed out in connection to inter-machine modeling activities [10]. These results that fueling via neutral beam injection is important in explaining the value of the density peaking in present experiments, lead to the question if, when fueling is not large, as expected in a reactor, the density profile could not be as peaked as observed in present machines. While that may hold for present experimental scenarios in which the parameters are such that fueling is important, this does not necessarily imply that the density peaking will be low or absent in a reactor. Instead, it simply means that one has to perform an experiment in which fueling is negligible, but still in reactor-relevant conditions. The peaking observed in such an experiment can then be taken as a proxy of what should be observed in a reactor with similar dimensionless parameters. It is shown here that the predicted peaking for a reactor is in agreement with theoretical expectations. In general, the density peaking arises via an interplay between transport mechanisms and the fueling source. It is not evident a-priori how to disentangle the two effects in a single experiment. This is what has been done in this work as it will be shown later.

A parameter that can conveniently describe the source-over-transport effect is 
$\left(S_{\text {str }}=\right.$ source strength $)$ :

$$
S_{\text {str }}=2000 \frac{P}{E n V} \frac{a^{2}}{\chi}
$$

where $P$ is the core NBI power in MW total injected power, $E$ the beam ions injection energy in $\mathrm{kV}, n$ the electron plasma density in $10^{19} \mathrm{~m}^{-3}, V$ the plasma volume, $a$ the plasma minor radius, $\chi$ the effective core heat transport diffusivity in $\mathrm{m}^{2} / \mathrm{s}$ (that is an average between the electron and the ion heat diffusivity). The factor 2000 is set such as to match the actual value from 1 ASDEX Upgrade case, where it assumes the value $S_{\mathrm{str}} \approx 0.1$ and where infact the source turns out to be negligible, as it will be shown later. This parameter can be compared between different machines, and, if much below unity, it means that the density profile is mostly determined by transport. Notice that the particle source provided by pellets is not included in the argument presented above. In present machines, pellet penetration can be substantial $[11,12]$, however it is expected that in a reactor the deposition location would be more periferal $[13,14]$. Nevertheless, it can only contribute positively to the actual density peaking. In this work, the effect of the additional pellet fueling is not adressed, but it can be added in the modeling and the effect on the density profile calculated.

An additional ingredient which is often ignored is the role of electromagnetic fluctuations, which become relevant at finite $\beta$. It is shown here that the predictions significantly change when going from electrostatic to electromagnetic turbulence. Although this effect is hard to extract from dedicated experiments, it has been found from multi-parameter regression in two existing machines [15].

This paper is organized as follows: section 2 describes the experimental setup and the resulting discharges, focussing on core transport properties. Section 3 discusses the modeling setup. Section 4 shows the modeling results and the physical interpretation in relation to the experiments. Section 5 discusses the extrapolation to a DEMO-like reactor. Section 6 draws the conclusions.

\section{Description of the experiments}

In these discharges performed on ASDEX Upgrade (AUG), the goal was to obtain the lowest possible core electron density (and collisionality) at fixed power, current, and field. Then, a combined density / NBI power scan is performed to check the effect of varying collisionality and particle source strength. The chosen engineering parameters for this discharge database are: $B_{\mathrm{T}}=2.5 \mathrm{~T}$ at $R=1.65 \mathrm{~m}$, and $I_{\mathrm{p}}=0.8$ MA, which leads to a $q_{95} \sim 5$. This is somewhat larger than the typical reactor parameters (e.g. ITER, DEMO) of $q_{95}$ closer to 3-4. The choice of keeping a lower current was justified by the priority of achieving low density. The magnetic field of $2.5 \mathrm{~T}$ has been chosen to profit of more common setup for concomitant operation of resonant magnetic perturbations (RMPs) and the ion cyclotron resonance heating (ICRH) antenna. Nevertheless, discharges at $1 \mathrm{MA}, 2.5 \mathrm{~T}$ were also performed, which have a $q_{95} \sim 4.5$, while discharges at $1 \mathrm{MA}$ and $2 \mathrm{~T}$ are planned for a future campaign. 


\begin{tabular}{|c|c|c|c|c|}
\hline & $\# 34400$ & $\# 34403$ & $\# 34405$ & $\# 34423$ \\
\hline$<n_{\mathrm{e}}>\left[10^{19} m^{-3}\right]$ & 3.8 & 5.2 & 6.7 & 5. \\
\hline$P_{\mathrm{NBI}}[\mathrm{MW}]$ & $2.5-1$ & $2.5-1$ & $2.5-1$ & 0. \\
\hline$P_{\mathrm{ICRH}}[\mathrm{MW}]$ & 2. & 2. & 2. & 4. \\
\hline$I_{\mathrm{p}}[\mathrm{MA}]$ & 0.8 & 0.8 & 0.8 & 0.8 \\
\hline$B_{\mathrm{T}}[\mathrm{T}]$ & 2.5 & 2.5 & 2.5 & 2.5 \\
\hline
\end{tabular}

Table 1. Global parameters for the sequence of shots performed on ASDEX Upgrade and studied in this work.

The heating scheme used was centrally deposited 2 or $4 \mathrm{MW}$ of ICRH, plus NBI injected in 4 power steps: $2.5,2,1.5$ and $1 \mathrm{MW}$. In addition, a pure-ICRH discharge has also been performed, with $4 \mathrm{MW}$ of ICRH applied. In each of the performed discharges, RMP coils [16] are used to maintain a low edge density, taking advantage of the so called pump-out effect [17]. The plasma density levels, which are varied on a shot-to-shot basis, are about (line average densities) $3.8,5.2$, and $6.710^{19} \mathrm{~m}^{-3}$. The pure ICRH case had a line average density around 5 . The shot numbers and parameters are summarized in table 1.

As regards the measurements used to analyze the experimental profiles, the ECE radiometer has been used for the electron temperature profile. The ion temperature profile is diagnosed by means of charge exchange recombination spectroscopy (CXRS), which relies on both the dominant NBI source, and an additional NBI source used for short (12 ms) beam blips. Both ion temperature and rotation data are then compared and found to lead to the same profiles. The electron density is diagnosed by means of the edge lithium beam diagnostics, while for the core both Thomson scattering and IDA reconstruction technique [18] are used for comparison. The data are then fitted to get smooth profiles especially around the chosen position for the boundary condition of the simulations. All the measured profiles are then time averaged, where the reference time of the 4 segments per each shot are $t=[3.39 ; 4.39 ; 5.405 ; 6.4] \mathrm{s}$, while the time intervals of averaging around these points are $d t=[0.01 ; 0.01 ; 0.025 ; 0.02]$ s. Finally, the safety factor profile and the plasma boundary shape are taken from the equilibrium reconstruction (no direct measurement of the safety factor was performed during these discharges).

By means of this double scan in density and NBI power, one has a simultaneous scan in collisionality and in core particle source level that can then be extrapolated to reactor-relevant parameters. Notice that electron cyclotron heating (ECRH) was specifically not used at these densities, as it would lead to rather large $T_{\mathrm{e}} / T_{\mathrm{i}}$ ratios, which are unrealistic when compared to the expectations for a reactor plasma.

Now, to be able to compare to an actual reactor design, it is recalled here the EUDEMO 2015 design [5]. Its parameters are compared to the present discharges in the density range 3.8-6.7 line average density, in table 2 .

As can be seen from the parameters, some of the strongest differences are in 


\begin{tabular}{|c|c|c|}
\hline & DEMO & AUG (low to high density) \\
\hline$\nu^{*}$ & $10^{-3}-10^{-2}$ & $10^{-2}-10^{-1}$ \\
\hline$\beta_{\mathrm{N}}$ & $2.4-3$ & $.8-1$ \\
\hline$Q_{\mathrm{i}} / Q$ & $0.6-0.65$ & $0.53-0.6$ \\
\hline$\rho^{*}$ & $\sim 10^{-4}$ & $\sim 10^{-3}$ \\
\hline$Q_{\mathrm{i}} / Q_{\mathrm{gB}}$ & $\sim 5$ & $\sim 10$ \\
\hline$q_{95}$ & $3.5-4$. & 5 \\
\hline$S_{\mathrm{NBI}} \tau_{\mathrm{E}} / N_{\mathrm{e}}$ & $\sim 10^{-2}$ & $\sim 10^{-2}$ \\
\hline$T_{\mathrm{NBI}} \tau_{\mathrm{E}} /\left(R M N_{\mathrm{e}} c_{\mathrm{s}}\right)$ & $\sim 10^{-3}$ & $\sim 10^{-2}$ \\
\hline
\end{tabular}

Table 2. Comparison of EU-DEMO 2015 (DEMO) parameters with present discharges. Local parameters $\nu^{*}, Q_{\mathrm{i}} / Q, \rho^{*}$, and $Q_{\mathrm{i}} / Q_{\mathrm{gB}}$ are evaluated at mid-radius.

collisionality, $\beta$, and safety factor. Of these three parameters, the one that is scanned the most is the collisionality, which helps in defining the trend going towards reactor parameters. The safety factor will be adjusted in future experiments performed at 1 MA and $2 \mathrm{~T}$, which will also help raising $\beta$. As an example, time traces and profiles of the lowest density discharge \#34400 are shown in figure 2.

To characterize the dependencies of the density peaking on the fueling source (NBI) and on intrinsic plasma parameters (e.g. collisionality), different plots are shown in figure 3. Notice that the collisionalty parameter is given as $\propto N / T^{2}$, where $N$ is the electron density and $\mathrm{T}$ the electron temperature at mid-radius. This parameter arises if one considers the Coulomb collisional factor $n / T^{3 / 2}$, normalized to the sound speed $\propto \sqrt{T}$. It is clear that the collisionality orders the normalized density gradient better than NBI power, the highest density peaking observed at the lowest collisionality. The role of the NBI source is negligible as observed from the first two plots. Moreover, the pure RF case shows an even higher peaking at the same collisionality. This appears to be due to the larger electron temperature peaking, suggesting that turbulence-driven thermodiffusion electron particle convection is relevant in these conditions.

Additional dependences not shown here could be on the magnetic safety factor and magnetic shear. However, since these are not measured, and these cases all have the same current, one expects the statistical variation of these parameters to be well inside the error bars of the equilibrium reconstruction. As such it is assumed that these cases work at "constant" magnetic shear and safety factor, which are then not chosen as parameters to be checked for eventual hidden dependences.

In figure 4 the behavior of the other normalized gradients is also shown. One can see that the electron temperature gradient decreases, whereas the ion temperature gradient increases, as the overall plasma density is increased (e.g. collisionality increases). This closely resembles the LOC-SOC transition behavior [19, 20, 21]. Infact, although these plasmas are in $\mathrm{H}$-mode, core physics has the same character as in $\mathrm{L}$-modes. 

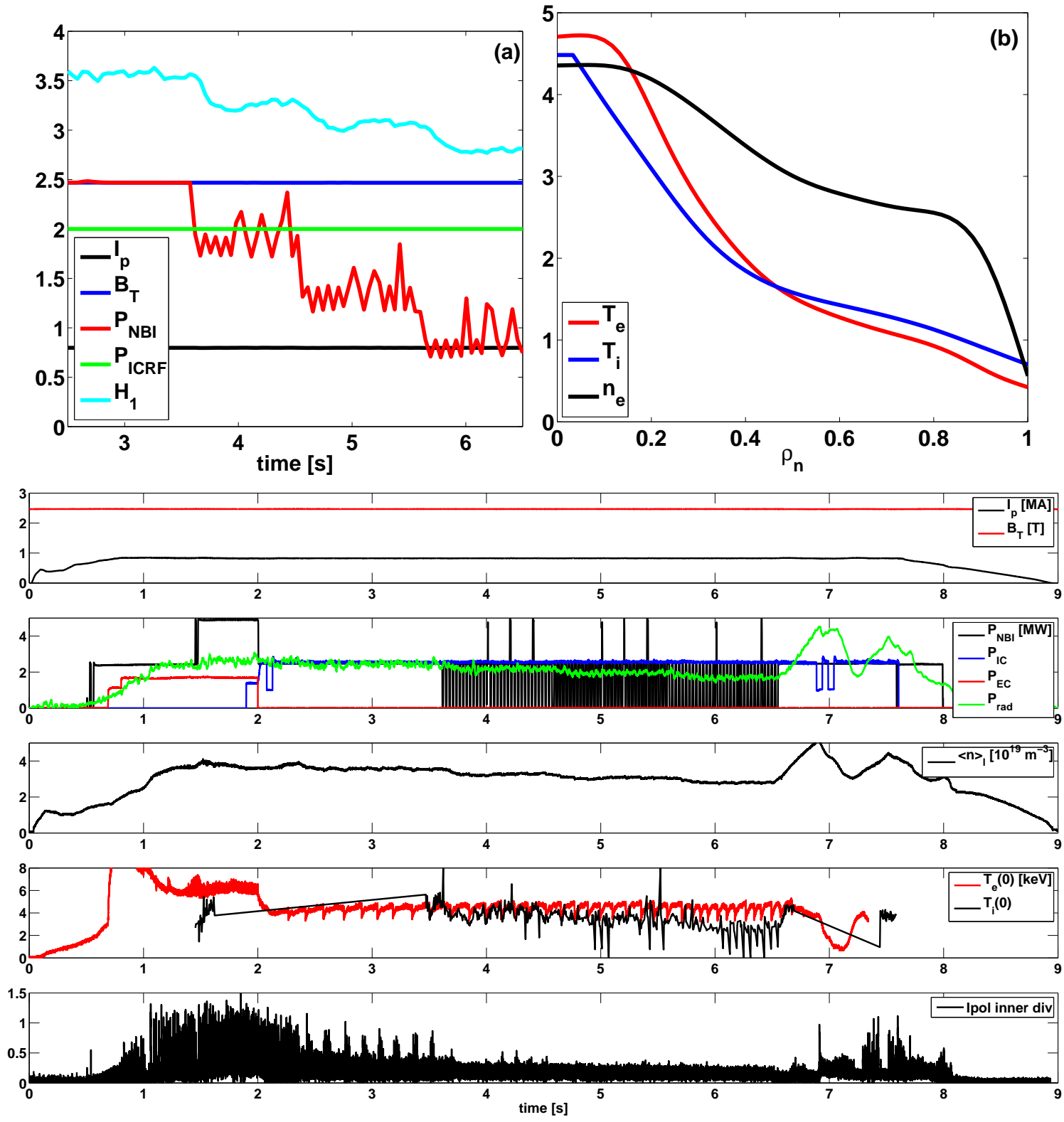

Figure 2. a, top) For the lowest density case \#34400: (top left) time traces of the plasma current $I_{\mathrm{p}}[\mathrm{MA}]$, magnetic field $B_{\mathrm{T}}[\mathrm{T}]$, NBI power $P_{\mathrm{NBI}}[\mathrm{MW}]$, ICRH power $P_{\mathrm{ICRH}}[\mathrm{MW}$, and line integrated density from interferometer chord H1. b, top) Electron temperature (red), ion temperature (red) and electron density (black) profiles as a function of $\rho_{\mathrm{n}}$ diagnosed at $t=4.5 \mathrm{~s}$; bottom) discharge overview including time traces of plasma current, magnetic field, power sources, line average density, central temperatures, innter divertor target poloidal currents. 

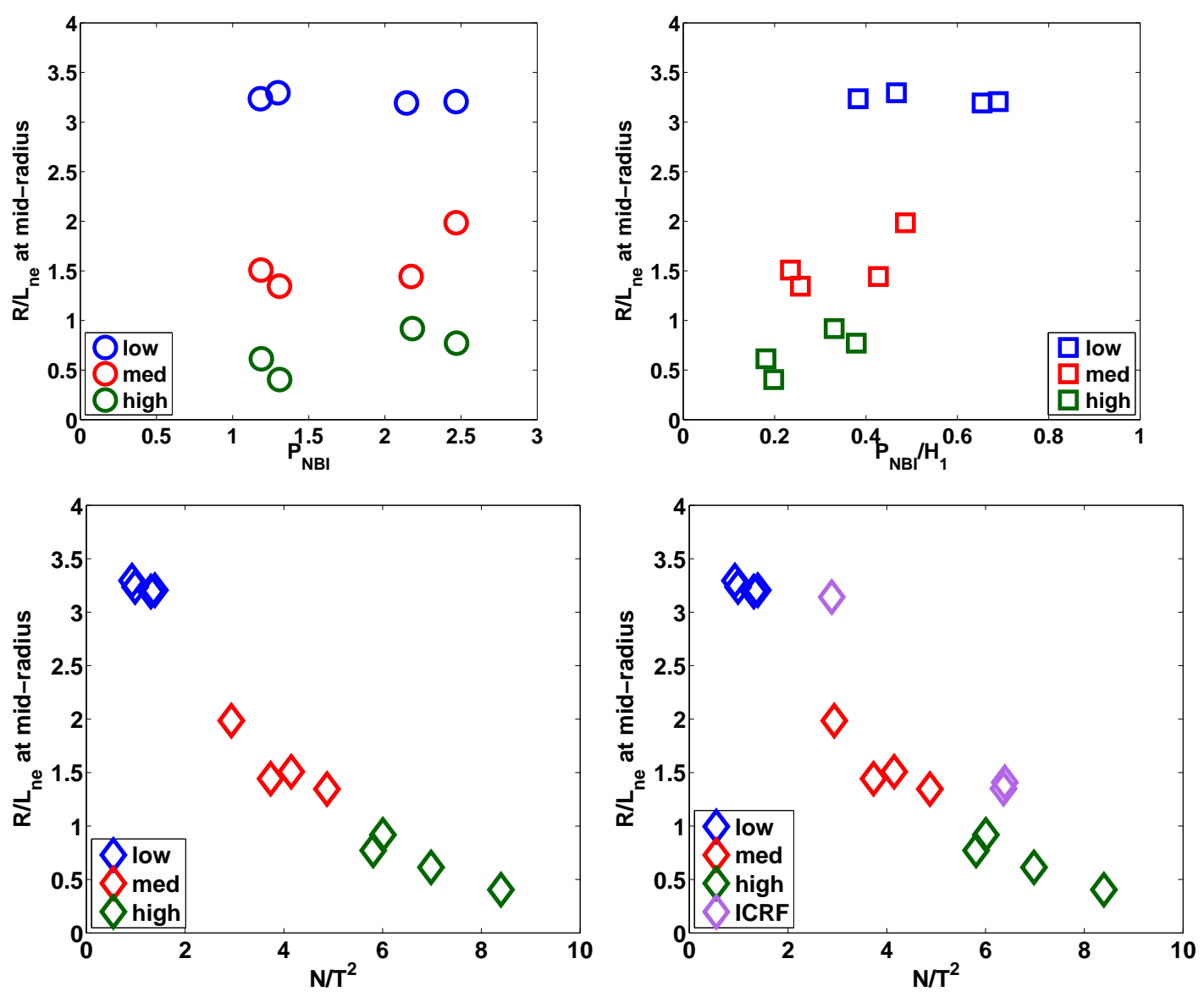

Figure 3. a) Normalized density gradient at mid-radius as a function of the NBI power for the three discharges at low, medium, and high density; b) same plot but using NBI power over line integrated density as ordinate; c) same plot but using density over temperature squared (e.g. collisionality) at mid-radius for the ordinate; d) same as c) but adding the pure-RF points.
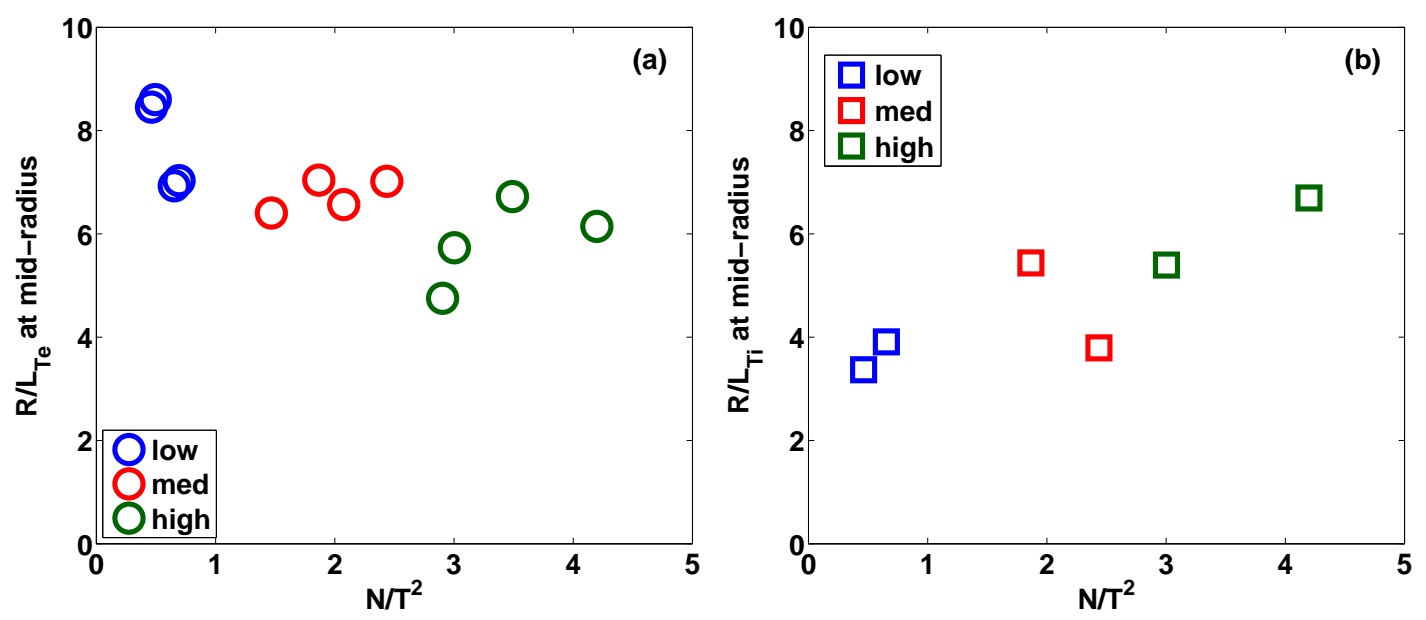

Figure 4. Normalized electron a) and ion b) temperature gradients at midradius as a function of the collisional parameter $n_{\mathrm{e}} / T_{\mathrm{e}}^{2}$. 


\section{Modeling setup}

The predictive runs, which employ the quasi-linear turbulence model TGLF [22, 23], are performed inside the ASTRA transport code [24, 25]. The boundary conditions for the evolution of the core electron and ion temperature and densities are set at 0.85 in the normalized toroidal flux coordinate. For $T_{\mathrm{e}}, T_{\mathrm{i}}$ the boundary condition is of fixed value, that is the experimental measured value at that position. For $n_{\mathrm{e}}$, the boundary condition at 0.85 is of particle flux equal to the core fueling source. The electron temperature, ion temperature, and electron density profiles are all evolved self-consistently according to the given power and particle sources. The current profile instead is fixed at the value provided by the equilibrium reconstruction. For the effective charge, a scaling of the type $Z_{\text {eff }} \propto 1 / n_{\mathrm{e}}^{2}$ is used, where the lowest density case has a $Z_{\text {eff }} \sim 2.5$, and the impurity is boron $(\mathrm{B} 5+)$.

Regarding the turbulence flux calculations, we use the multi-scale saturation model (sat1) option in TGLF [26]. Rotation effects (ExB perpendicular shearing and paralllel velocity gradients) are set to 0 . Also fast ion effects are neglected, that is no fast ion species is included in TGLF. While rotation is not expected to be relevant in these conditions (too low torque input), the fast ions could instead lead to some effects on turbulence, due to their large presence [27, 28]. Neoclassical transport is calculated using NCLASS [29]. It is found that neoclassical transport makes up for $20 \%$ at maximum of the total ion energy transport.

The effect of macroscopic MHD, that is the sawtooth crash, is not simulated self-consistently, but an artificial energy and particle diffusivity is added on top of the turbulence-driven and neoclassical ones. This artificial diffusivity is added in the sawtooth-affected area and basically it keeps the profiles flat inside that area. The sawtooth inversion radius for the simulated cases is roughly 0.2 in normalized radius. Inside this radius, we have applied an additional $0.5 \mathrm{~m}^{2} / \mathrm{s}$ diffusivity to all channels to mimic the effect of sawteeth.

The heating and particle sources are computed in the following ways. Since the boundary condition for the profiles calculation is set well inside the pedestal, it is assumed that the neutral particle source is absent in the simulation core. The fueling from NBI is computed consistently with the heat deposition using the NBI module available in ASTRA [30]. Ohmic heating and energy equipartition between electrons and ions are both computed self-consistently. ICRH heating is simplified and represented as a Gaussian profile centered at $r / a=0.05$ and of width $d r / a=0.15$, where the total integrated power is equal to the assigned power (2 MW for all cases with NBI, $4 \mathrm{MW}$ for the pure ICRH case). The split of power deposition between electrons and ions is $70 \%$ to the electrons and $30 \%$ to the ions. This is consistent with estimates obtained for \#34400 using TORIC-SSFPQL [31]. The radiated power is defined as being composed of brehmsstrahlung, syncrotron, and line radiation dominated by tungsten (W). W concentration is assigned as $8 \cdot 10^{-4} / n_{\mathrm{e}}$ for all cases, so that $\mathrm{W}$ density is effectively 

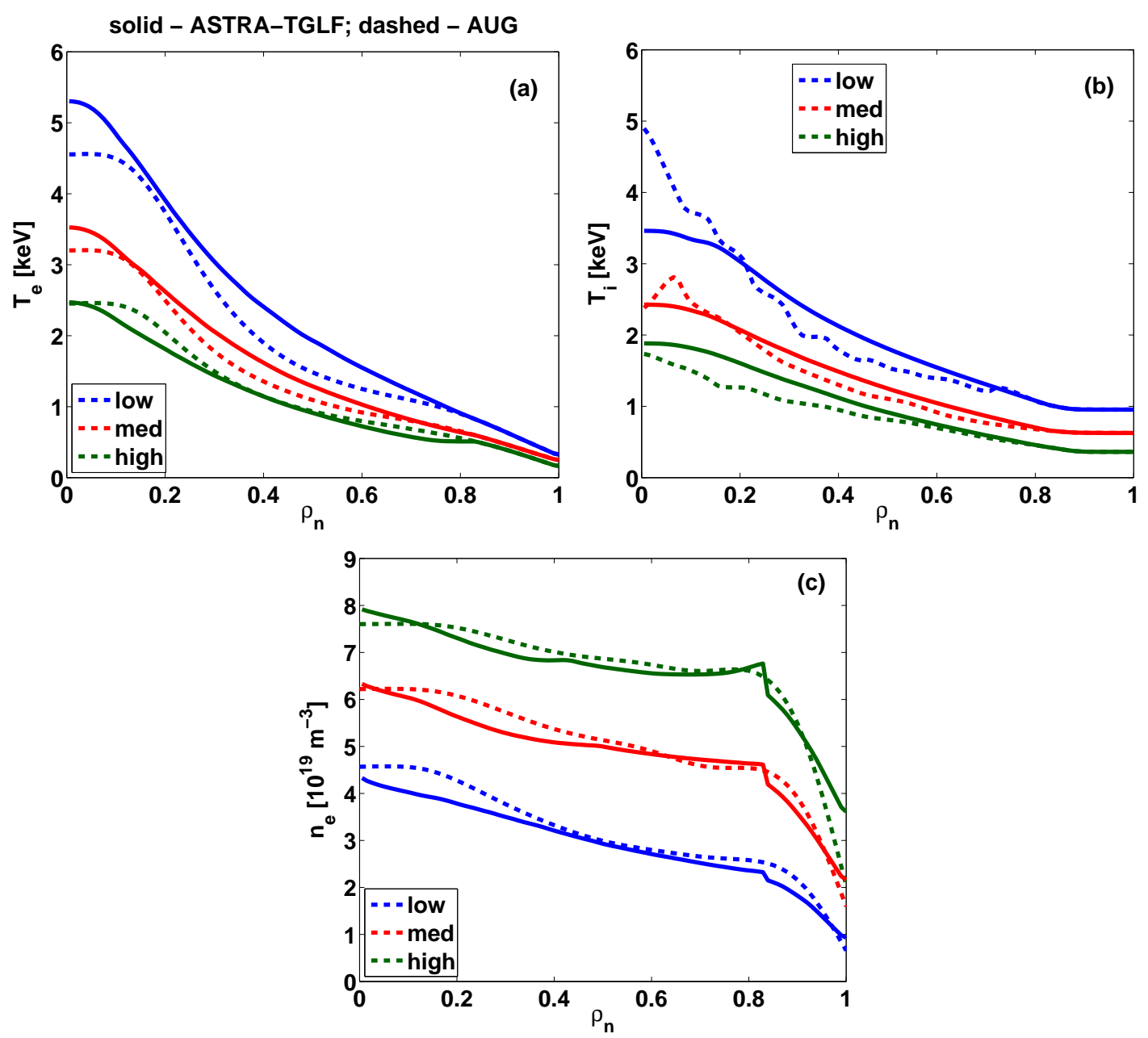

Figure 5. Comparison of simulated (solid) and experimental (dashed) profiles of electron temperature (a), ion temperature (b), and electron density (c). The discharges at different densities are indicated in the legend as "low", "med", and "high" density.

$8 \cdot 10^{-4}$ and it is constant in radius. This value leads to a total radiated power which is in the same ballpark as the experimental observation for the analyzed cases.

\section{Modeling results}

The cases chosen for modeling are: the time point is $t=4.39 \mathrm{~s}$, where the NBI power is the same 2 MW for all cases, for discharges \#34400 ("low" density), \#34403 ("med" density) and \#34405 ("high" density).

The results of the modeling are given in figure 5 for the three profiles $\left(T_{\mathrm{e}}, T_{\mathrm{i}}, n_{\mathrm{e}}\right)$. In general, the agreement is rather good, although for the low density case the core gradients are underestimated, especially in $T_{\mathrm{i}}$. This could be due to the neglect of additional stabilizing mechanisms (NBI-driven toroidal rotation, NBI+ICRH-generated 


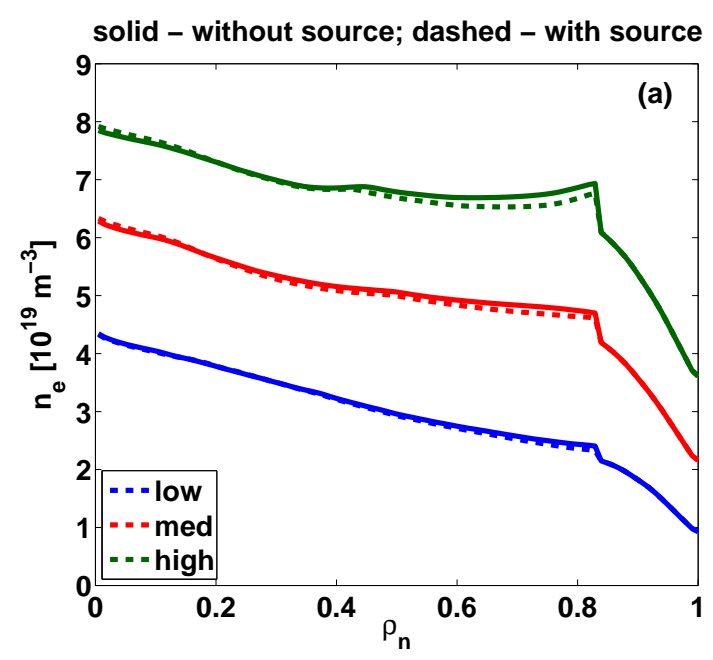

Figure 6. Comparison of simulated electron density profiles with (dashed) and without (solid) the NBI particle source.

fast ions).

For the density profile specifically, the trend of reducing the peaking is reproduced, with a flattened region between 0.4 and 0.8 , due to the increased role of outward convection proportional to the local collisionality [32, 33].

The result presented previously, are now compared in figure 6 with cases run at null core particle source. This confirms that the density profile is determined almost solely by turbulence. As one can see, the profiles are hardly modified (a very small decrease in peaking is noticeable). This is in agreement with the estimate done in table 2 , which shows that the source is expected to be negligible. To check when the source starts to play a role, for the intermediate density case \#34403, we have performed simulations where we multiplied the source amplitude by factors of $0^{*}, 1^{*}$, $2^{*}, 3^{*}, 6^{*}$ and $10^{*}$. The results show that the values of the central density are respectively $5,8,13,24,40 \%$ more than the no-source case for $1^{*}, 2^{*}, 3^{*}$, $6^{*}$ and $10^{*}$ times the actual source.

\subsection{Analysis of turbulence properties}

It is instructive to look at the turbulence spectra for the previously simulated three density cases. The spectra are obtained from the steady-state converged ASTRA-TGLF runs, that is consistent with the predicted profiles.

In figure 7 the spectra of the growth rates (normalized to the maximum value) and of the phase velocity of the turbulence are portrayed. It is evident that there is more substantial intermediate-k TEM contribution in the low density cases, which disappears in the medium and high density cases. The latter two have similar spectra. In figure 8, the spectra of the electron and ion heat fluxes are displayed. Both heat fluxes are driven mostly by the low-k part of the spectrum (for the ions this is the only driving 

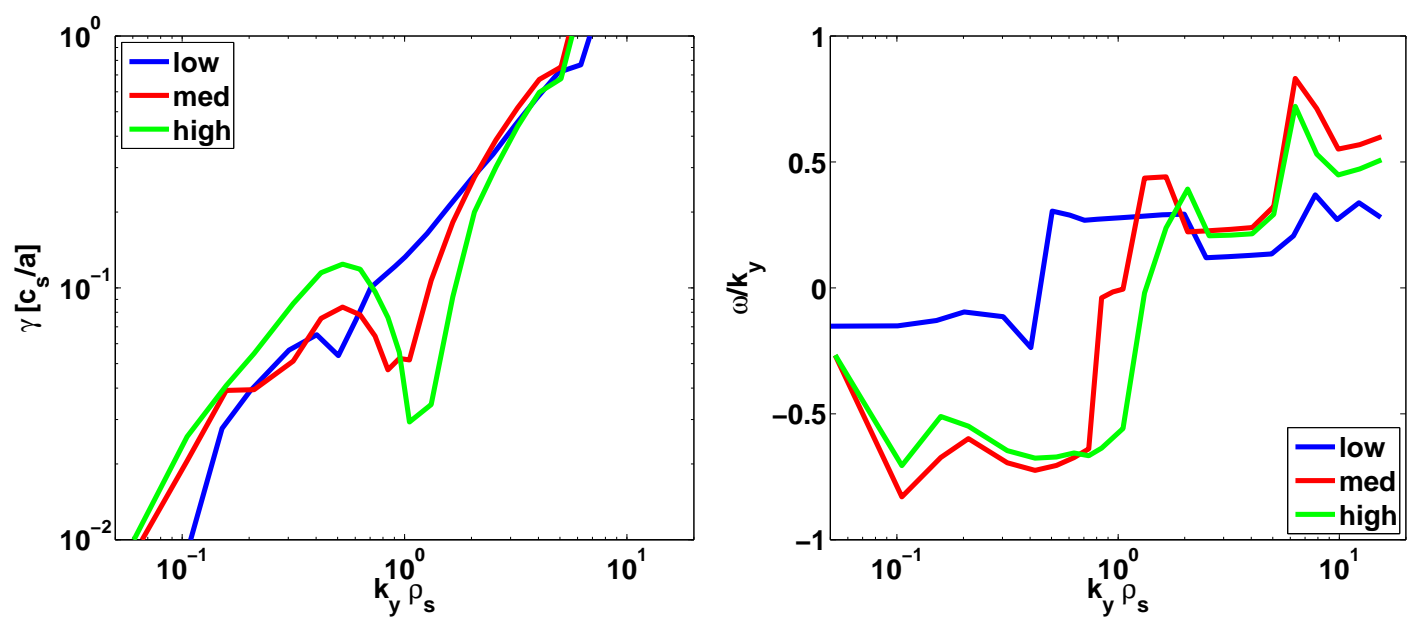

Figure 7. a) Growth rate of the most unstable mode as a function of the binormal mode number $k_{\mathrm{y}} \rho_{\mathrm{s}}$, calculated at mid-radius and for the three densities. b) Phase velocity $\omega / k_{\mathrm{y}}$.
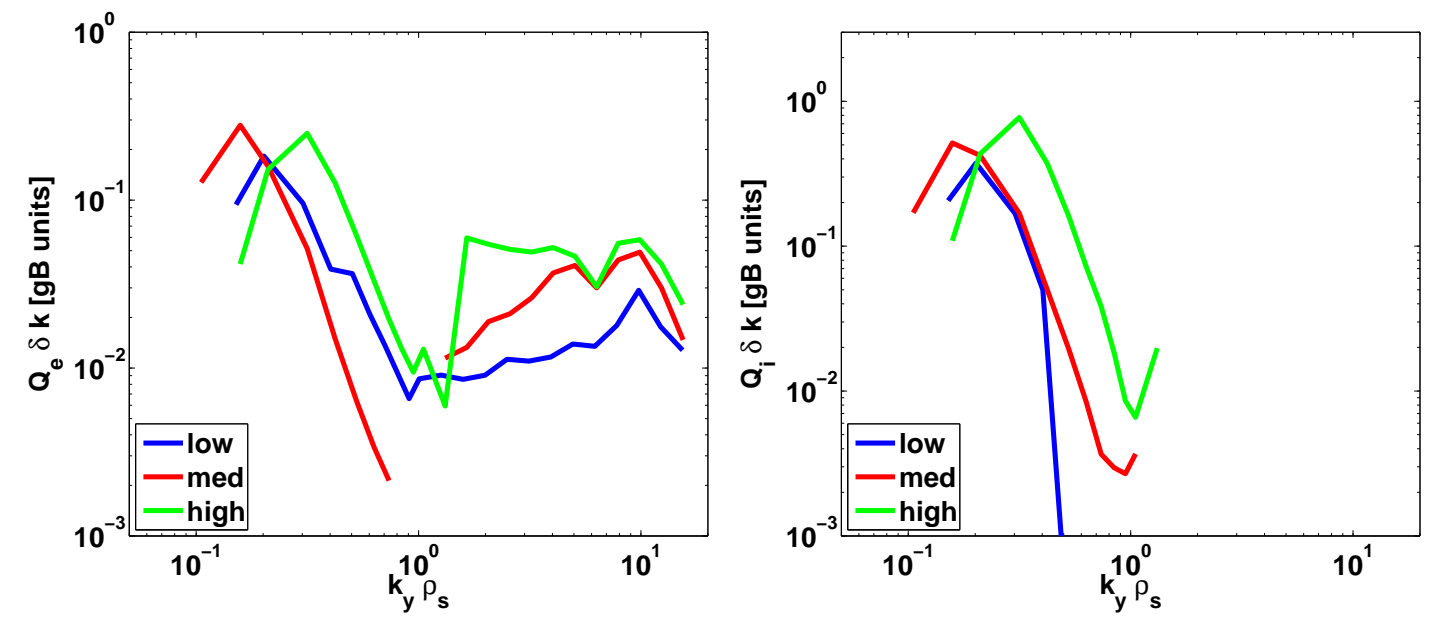

Figure 8. Spectra of electron (a) and ion (b) heat fluxes as a function of the binormal wavenumber.

region since at intermediate-to-high-k they behave "adiabatically" due to the Larmor radius averaging), but it is interesting to observe an increasing high- $\mathrm{k}$ component when the collisionality increases. This is due to several features: $T_{\mathrm{e}} / T_{\mathrm{i}} \rightarrow 1$, lower impurity content, and flatter density, all leading to more ETG activity.

The ion heat flux is also continuously increasing going from lower to higher densities, in accordance with the observation that $R / L_{T i}$ increases monotonically due to more effective collisional temperature coupling from the electrons to the ions. 


\section{Extrapolation to ITER and DEMO-like parameters}

From the results presented in the previous section, one could deduce that the extrapolation towards ITER or DEMO-like parameters would be feasible using the same modeling setup, upon replacing the machine geometry, auxiliary and radiated power, and values at the boundary conditions location. The ITER-like case, in terms of design parameters, is based on reference [34], while the DEMO-like on reference [5].

For the latter, the temperature is chosen as $T_{\text {ped }}=[4.5,5.5] \mathrm{keV}$ respectively for the ITER-like and the DEMO-like case. For the electron density profile, the boundary condition at the pedestal top is defined by fixing the pedestal top Greenwald density fraction as 0.55 for the ITER-like case and at 0.85 for the DEMO-like case. This choice leads to very similar absolute density values (since in the ITER-like case the ratio $I_{\mathrm{p}} / a^{2}$ is almost as twice as large as in the DEMO-like case).

The plasmas are in these cases defined as composed by $50 \% \mathrm{D}$ and $50 \% \mathrm{~T}$. The power coming from fusion reactions is then computed and added to the heating sources self-consistently. Additional considered ion species are He and Xe. However, to make the simulations lighter, these 2 are combined in an "average" impurity which charge and mass are such as to make the effective charge $Z_{\text {eff }}$ of order 1.3 .

Auxiliary power (assumed split 50-50 between electrons and ions) is given in total as $50 \mathrm{MW}$ for both the ITER-like case and the DEMO-like case. In both scenarios the particle fueling source is neglected. Nevertheless, it must be noticed that, apart from the small component of NBI fueling, there could be an important contribution via pellet fueling, which would be peripheral $(r / a>0.7)$, but would positively contribute to the density peaking on top of the prediction given in this work. Modeling including the pellet source is left for future work.

Regarding the current density profile and the safety factor, one must discuss the position of the sawtooth inversion radius and the role of sawteeth. In the present work, it is assumed that the safety factor profile is relaxed, since it is known that in a reactor scenario the sawtooth stabilization via alpha particles would lead to very long sawtooth periods $[35,36]$. As such, although the sawtooth inversion radius for ITER-like and DEMOlike plasmas would be roughly between 0.4 and 0.5 in normalized radius, we ignore its effect.

The plasma shape for these cases is given in terms of three moments. The following values for the shape parameters are used, respectively for the ITER-like and the DEMOlike: major radius $R=[6 ., 9.] \mathrm{m}$, minor radius $a=[2 ., 3.] \mathrm{m}$, elongation $k=[1.65,1.7]$, triangularity $\delta=[0.36,0.4]$. Plasma current $I_{\mathrm{p}}=[15 ., 19.] \mathrm{MA}$, magnetic field at $\mathrm{R}$ $B_{\mathrm{T}}=[5.3,5.8]$.

\subsection{Profiles prediction}

The profile predictions for ITER-like and DEMO-like scenarios are shown in figure 9. A few interesting observations can be drawn from these simulations: 1) the ion and electron 

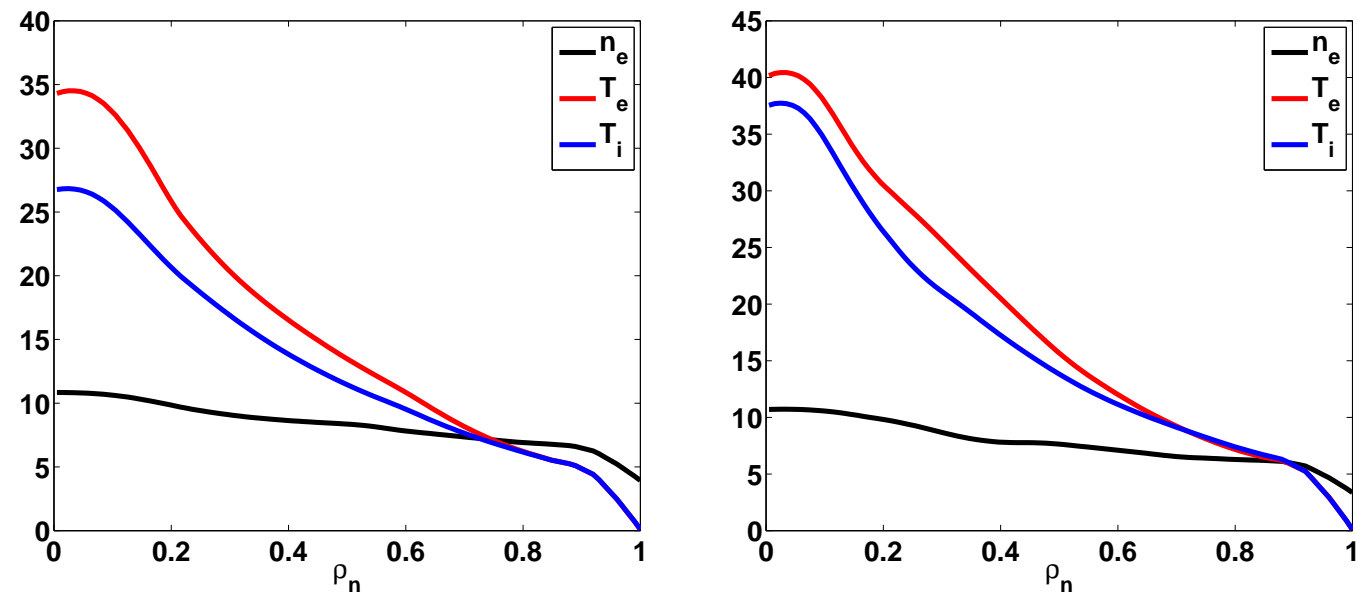

Figure 9. left) predictions for ITER ; right) predictions for DEMO.
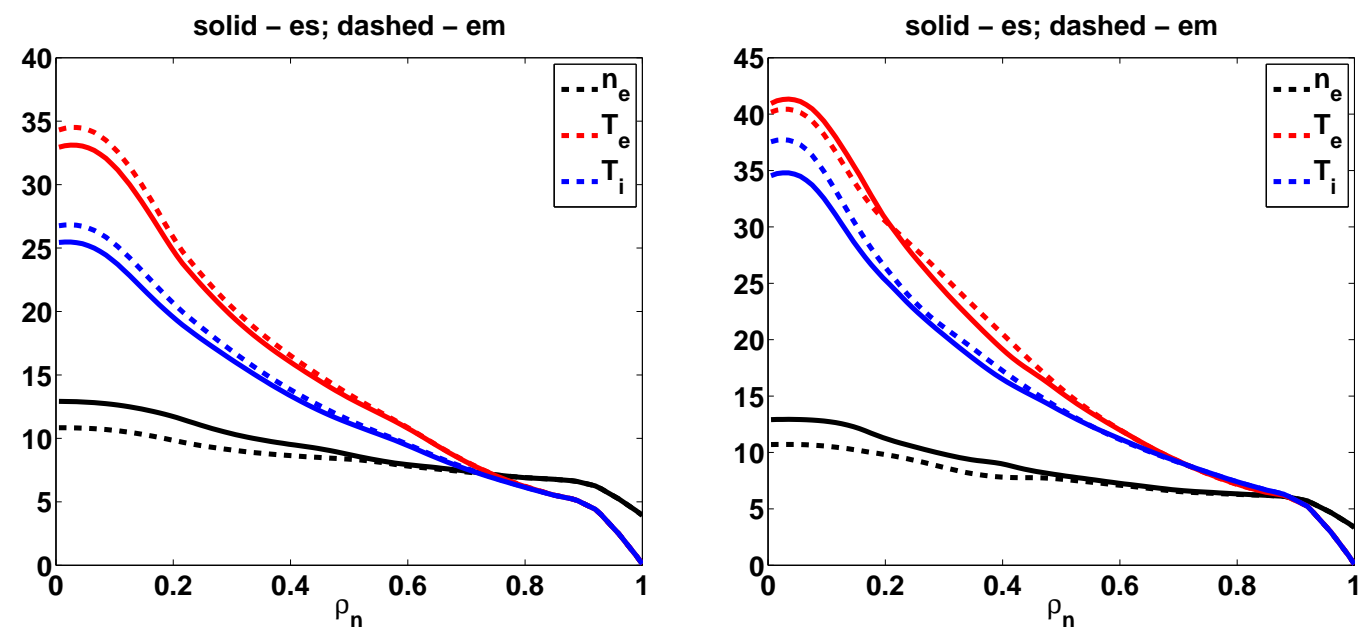

Figure 10. For the predictions of figure 9, comparison of runs obtained with (dashed), and without (solid) electromagnetic effects on the microinstabilities.

temperatures are predicted to be closer in DEMO, both because of the behavior of the alpha power partition between ions and electrons, plus the fact that the equipartition time is shorter compared to the confinement time with respect to the ITER scenario; 2) the density profiles are very similar between the two machines; 3) the overall density peaking factor is around 1.4 for both, consistently with previous studies [37].

Now, before considering the comparison with the ASDEX Upgrade cases, one can ask, why is the peaking lower than the one obtained in ASDEX Upgrade experiments, even though the collisionality is higher. The answer is in the electromagnetic response of turbulence, which can be seen in figure 11. In the latter figure, the simulations are also performed in the electrostatic limit, i.e. setting $\beta=0$ in TGLF. It can be seen that the density is more peaked in the electrostatic case in both cases. On the other hand, the temperatures increase inversely proportional to the decrease of the 


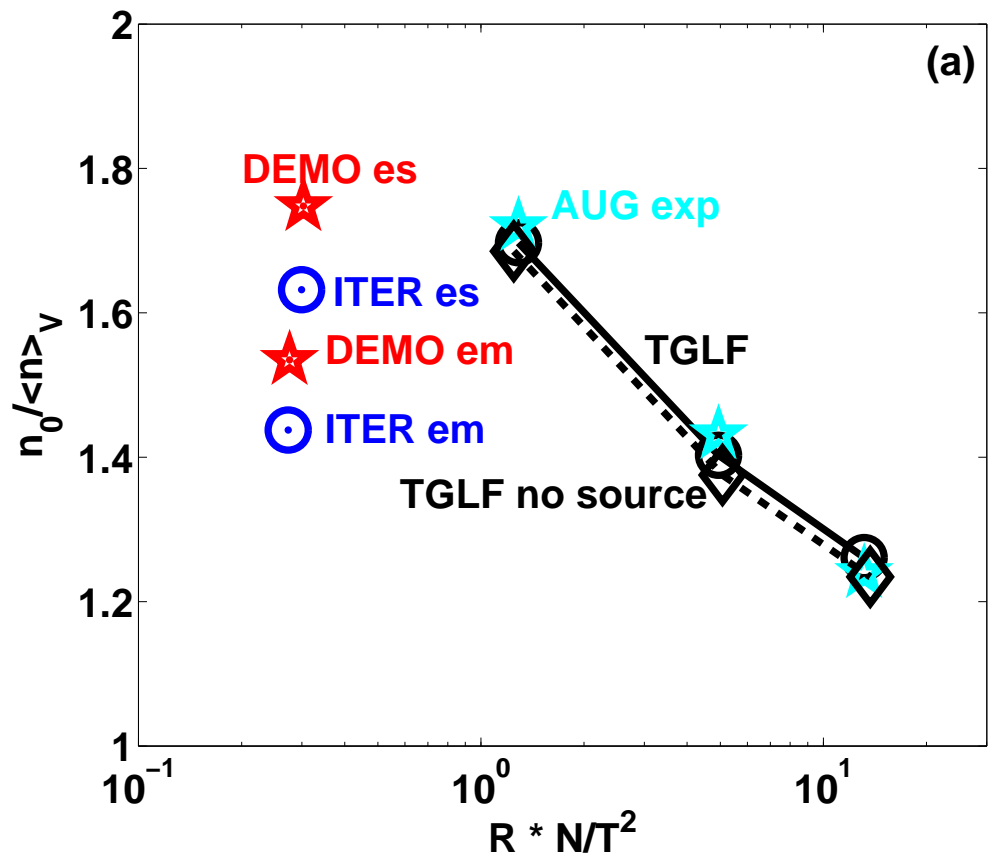

Figure 11. Comparison between ASDEX Upgrade predictions and ITER/DEMO-like predictions. The density peaking is plotted as a function of the inter-machine collisional parameter $R n_{\mathrm{e}} / T_{\mathrm{e}}^{2}$.

density. However this does not come from improved energy confinement with $\beta$, but simply from energy conservation. This effect is known from the theory standpoint and documented in the literature $[39,40]$. To have a global overview of why this is the case, one can look at figure 11. The peaking factors of the simulated cases are shown, as a function of the plasma collisionality parameter $R n / T^{2}$. The ASDEX Upgrade predictions are well ordered by this parameter, and the ITER/DEMO predictions follow on the predicted trend when running in the electrostatic limit. Notice that, having ITER and DEMO cases different shapes and different magnetic properties (e.g. magnetic safety factor, magnetic shear), the extrapolation based solely on collisionality does not directly apply (if one would use a regression). However, the values of these parameters are well inside the confidence range of the applied model (TGLF).

However, when electromagnetic fluctuations are taken into account, the peaking is reduced, and one gets the value observed in the simulations. As an interesting exercise, we have also run the DEMO-like case with pure D instead of DT, as species for TGLF. That is, fusion power and plasma mass and density is still calculated as if it was DT, but in TGLF the ionic species is D only (which is used to rescale the electron mass). The impact on the density peaking is the following: EM-DT $\rightarrow 1.53$, ES-DT $\rightarrow 1.77$, EM-DD $\rightarrow 1.54$, ES-DD $\rightarrow 1.78$. The results show that EM effects on the density peaking are a bit stronger for DT, due to the relative higher mobility of electrons on broken 

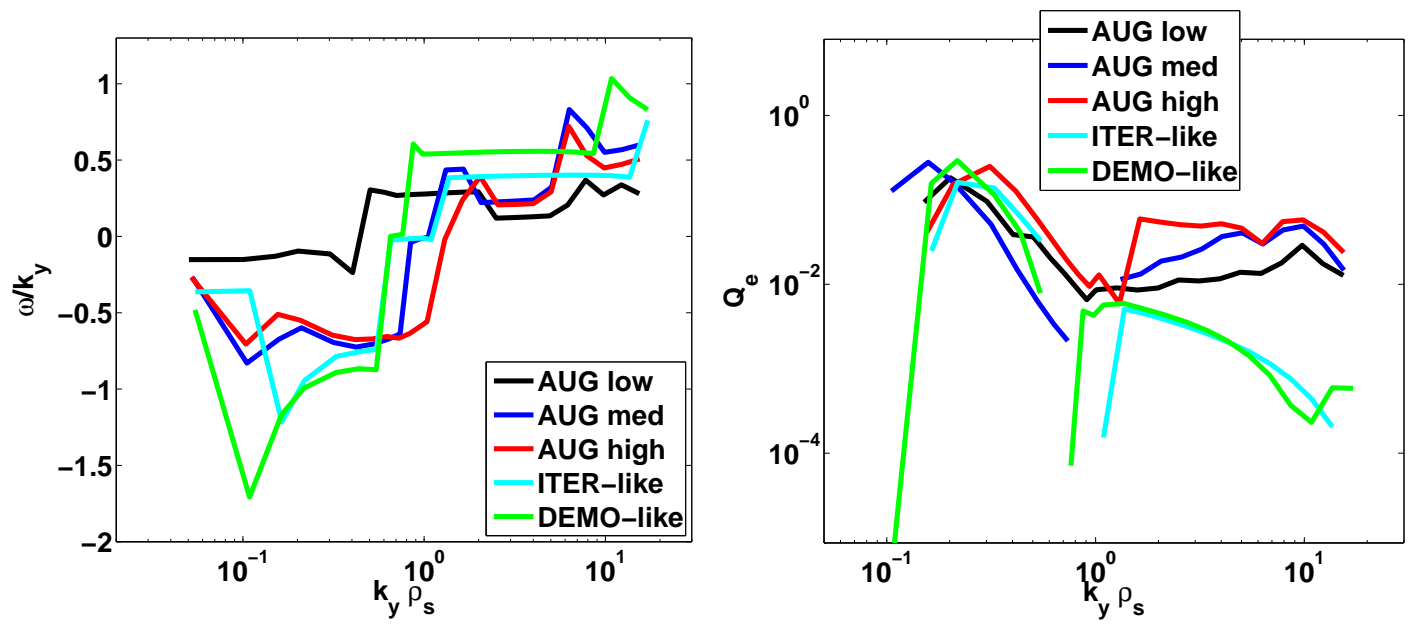

Figure 12. Comparison of spectra for the mode phase velocity (a) and the electron heat flux (b) where all three AUG density scan cases plus the ITER and DEMO predictions are collected.

field lines. In the ES case, the increased peaking in DD as compared to DT is due to the slightly increased temperature gradient since the favourable $\mathrm{gB}$ scaling. Of course this effect is present also in the EM cases. Nevertheless, the differences are very small between the two isotopes.

The reason why this is not observed in the ASDEX Upgrade discharges is seen in the last plot of figure 14, which shows the values of $\beta_{\mathrm{e}}$ at mid-radius. In the ASDEX Upgrade cases, this parameter takes values which are a factor 4 below the predicted values for a reactor-like machine.

\subsection{Analysis of turbulence properties}

As done in the previous section, one can look at the spectra of turbulence for the predicted ITER and DEMO-like machines, and compare them to the ASDEX Upgrade results. This is done in figure 12, where the phase velocity and the electron heat flux spectra are displayed, for the three AUG cases and the two reactor machines.

One can immediately see that the turbulence state in an almost collisionless reactor is actually closer to the medium collisionality AUG case, however, the heat flux spectrum is missing the high- $\mathrm{k}$ component.

There are several reasons why the reactor spectrum is less TEM dominated than the AUG case, despite the much lower collisionality (and identical aspect ratio): 1) the density profile is less peaked due to the electromagnetic effects; 2$) T_{\mathrm{e}} / T_{\mathrm{i}}$ is closer to 1.1-1.2 in the reactor prediction, while the AUG cases sits at around 1.4. These two elements make the turbulence more ITG-like at low-k.

At high $-\mathrm{k}$ instead the missing ETG contribution is due to the collisionality, i.e. in AUG, due to the higher collisionality, TEMs are practically absent and the ETG 


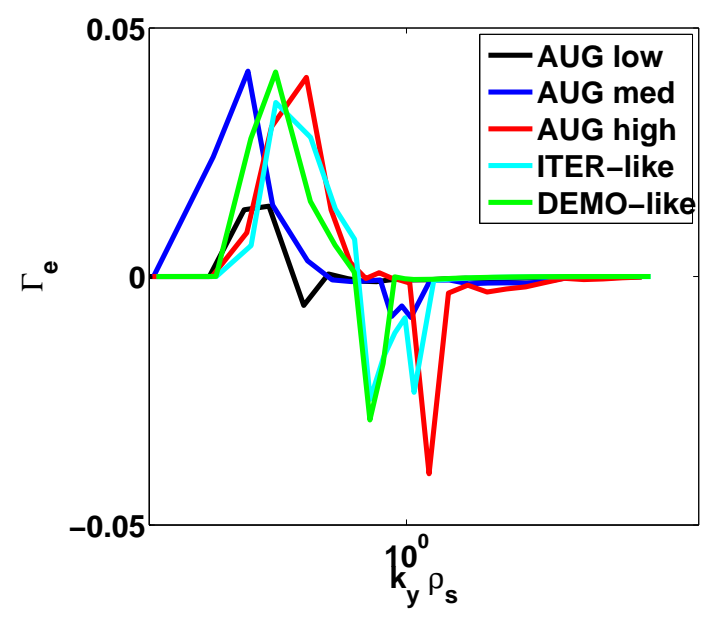

Figure 13. Comparison of spectra for the electron particle flux.

becomes a substantial contribution to electron transport. In a reactor-like scenario, the TEM is still important, although subdominant to the ITG. These results are consistent with analysis shown in [38].

In figure 13 we also show the particle flux spectra comparing AUG and reactors cases. It can be seen that the spectra peaks follow the heat flux spectra, while the signs point out a balance between low $-\mathrm{k}$ outward flux and intermediate to high-k inward flux, whereas for the lowest AUG density case the negative vs positive peaks are more balanced.

\subsection{A general "core-specific" figure of merit to compare present experiments and} reactor scenarios

It is useful to introduce a figure of merit to allow the comparison with present experiments, for core confinement quality:

$$
F_{\text {merit }}=\frac{1}{V_{b}}\left[\int_{0}^{b} \sigma_{\mathrm{DT}} n_{\mathrm{e}}^{2} d V\right]\left(\frac{1}{\left[n_{\mathrm{e}}\right]_{b}}\right)^{2} \frac{1}{\left[\sigma_{\mathrm{DT}}\right]_{b}}
$$

where

$$
\sigma_{\mathrm{DT}}=\sigma\left(\frac{T_{\mathrm{i}}}{\left[T_{\mathrm{i}}\right]_{b}}\left[T_{\mathrm{i}}\right]_{b}^{\text {reactor }}\right)
$$

All the parameters refer to the actual experiment, while $\left[T_{\mathrm{i}}\right]_{b}^{r}$ is the actual value of a reactor machine. The index $b$ refers to the boundary point (e.g. $r / a \approx 0.8$ or the pedestal top value). In practice this figure of merit represents how much fusion power a plasma scenario would produce if the profiles would be self-similar to those obtained in the present experiment, rescaled to the foreseen pedestal top values. So, of course, it is not a global figure of merit (because the pedestal is assigned), but it clearly defines the quality of core confinement and how well it would perform in a reactor to produce fusion power. 


\subsection{Comparing the figure of merit between ASDEX Upgrade and the reactor scenarios.}

Looking now at the values for formula 2, and comparing the simulations performed for ASDEX Upgrade with the reactor scenarios, in figure 14 one can see its behavior over different choices of parameters.

One can conclude that the $T_{\mathrm{e}} / T_{\mathrm{i}}$ ratio obtained in the ASDEX Upgrade discharges is still larger of what is needed to reach reactor-like parameters. However this is due, from the point of view of the actuator, to the mismatched value of $Q_{\mathrm{i}} / Q$, especially for the lowest AUG collisionality.

It is clear to see this collisionality dependence of the figure of merit, which is mainly due to the density profile peaking and temperature profile peaking.

Finally, the electromagnetic parameter $\beta_{\mathrm{e}} \propto n_{\mathrm{e}} T_{\mathrm{e}} / B^{2}$ is a factor of $3-4$ lower in AUG than in the reactor scenarios. This is mainly due to the difficulty of matching at the same time normalized heat fluxes (with respect to the gyro-Bohm level) and $\beta$, if one increases the power. Our goal in the planned discharges for the next campaigns is to reduce $\mathrm{B}$ to $2 \mathrm{~T}$ and try to match as best as possible both parameters.

As a side note, it is noteworthy that DEMO has a higher figure of merit than ITER. This is due to the ion logarithmic temperature gradient being larger in DEMO. The reason is that for DEMO, the partition of alpha power to the ions is larger than in ITER (that is, ITER is more electron heating dominated than DEMO). Note that this is also a consequence of the ion stiffness of the model employed, TGLF. If one would use another model with more sever ion temperature stiffness, the result would be different. However it is known from ASDEX Upgrade experiments as well that the temperature ratio is very sensitive on the ion to electron heat flux ratio [41].

\section{Conclusions}

In this work new experimental results have been presented, which aim at reaching reactor-relevant discharge parameters with respect to core transport properties. The discharges performed in ASDEX Upgrade are then modeled and interpreted with theorybased tools and the results used for extrapolation to actual reactor-like scenarios.

Attempts at modeling the discharges with the theory-based turbulence model TGLF resulted in a deeper understanding of the direction transport mechanisms take when decreasing the collisionality in standard $\mathrm{H}$-mode like conditions, at constant injected power.

Comparing dimensionless parameters in these discharges with reactor-like parameters, one is able to identify the major differences and explore the impact on the physics consequences for profiles prediction. By comparing spectra and fluxes obtained in TGLF between DEMO-like parameters and the lowest collisionality discharge obtained during this study, one has identified a few key parameters that will alter the profile shape when extrapolating to a reactor. In particular, the effect of collisionality itself, but also of electro-magnetic parameter $\beta$ have been identified. In a follow-up 

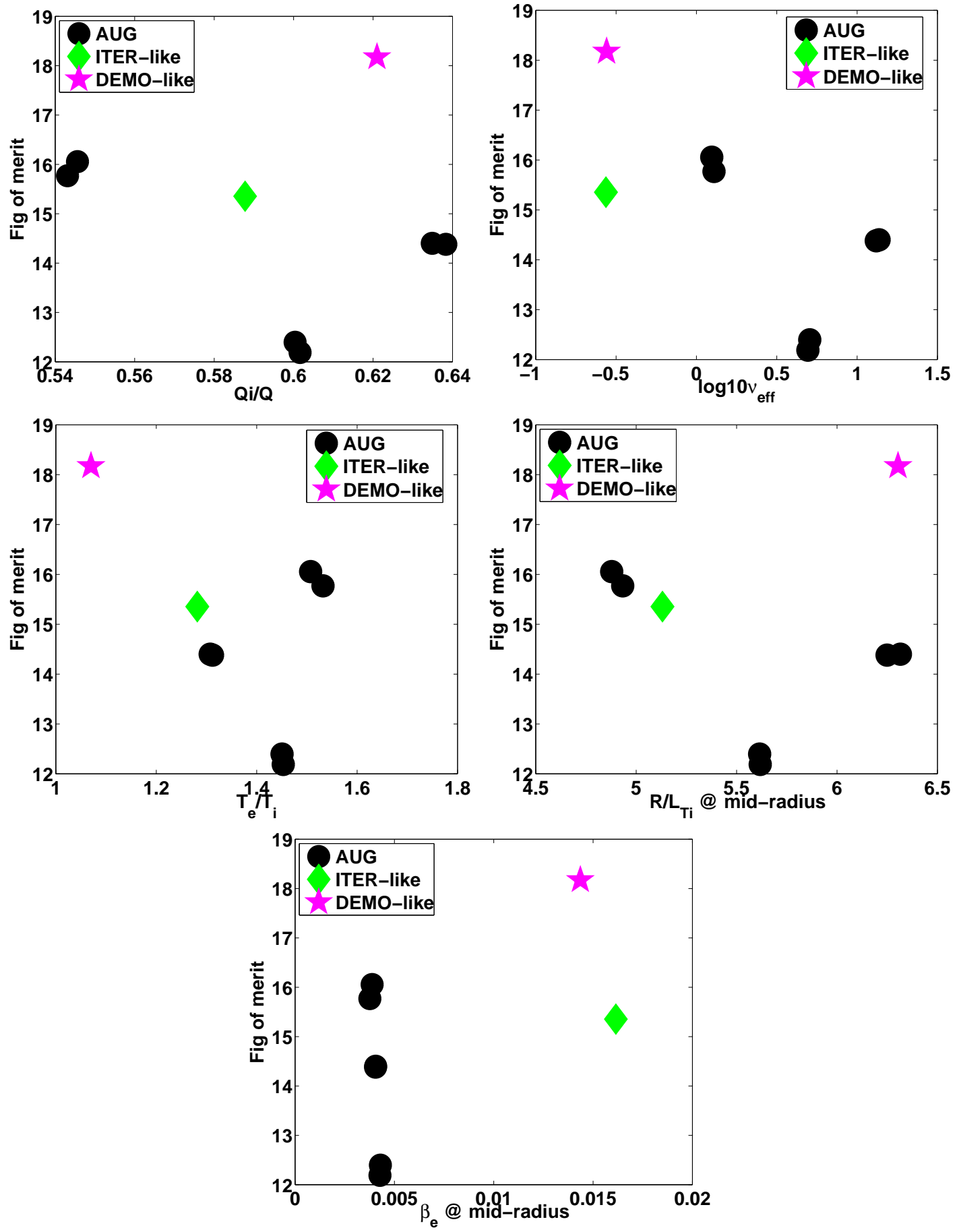

Figure 14. Comparison of the figure of merit defined in equation (2) of different cases w.r.t. 5 parameters: the ion to total heat flux ratio $Q_{\mathrm{i}} / Q$ taken at midradius, the effective collisionality $\nu_{\text {eff }}$ taken at mid-radius, the temperature ratio $T_{\mathrm{e}} / T_{\mathrm{i}}$ taken on axis, the ion temperature logarithmic gradient $R / L_{\mathrm{Ti}}$ taken at mid-radius, and the electromagnetic parameter $\beta_{\mathrm{e}} \propto n_{\mathrm{e}} T_{\mathrm{e}} / B^{2}$ taken at mid-radius. 
work, the role of the safety factor and possibly increasing $\beta$ in the AUG discharges will be studied.

Since there is a strong link between electron temperature and electron density, it is then proposed that the only meaningful way to increase the density peaking in a reactor is to increase the electron temperature gradient in the confinement region.

As a final note, one can make the argument that the exploitation of a higher $\beta$ may be best suited for advanced scenarios in which the temperature profiles also respond in a strong fashion by improving the confinement, which would overcome the loss of fusion power due to the reduced peaking with respect to the electrostatic limit. Another possibility in the baseline standard $\mathrm{H}$-mode scenario is increasing the field at constant power and radius, which would also lead to a lower $\beta$ and higher density peaking. These speculations are anyway part of the effort of finding the optimal operating point of a fusion reactor with input from theoretical considerations [42].

\section{Acknowledgments}

The Authors are grateful for the help of M. Teschke in setting up the RMP coils. This work has been carried out within the framework of the EUROfusion Consortium and has received funding from the Euratom research and training programme 2014-2018 and 2019-2020 under grant agreement No 633053. The views and opinions expressed herein do not necessarily reflect those of the European Commission.

\section{References}

[1] F. Romanelli et al., European Fusion Development Agreement (EFDA) (2012), ISBN 978-3-00040720-8

[2] G. Federici et al., 2017 Nucl. Fusion 57092002

[3] W. Biel et al., Fus. Eng. Design, Volume 123, November 2017, Pages 206-211

[4] E. Fable et al.,Fus. Eng. Design, Volume 130, May 2018, Pages 131-136

[5] R.P. Wenninger et al 2014 Nucl. Fusion 54114003

[6] R. Wenninger et al 2015 Nucl. Fusion 55063003

[7] E. Fable et al 2017 Nucl. Fusion 57022015

[8] L. Garzotti et al 2006 Nucl. Fusion 46994

[9] M. Valovic et al 2007 Nucl. Fusion 47196

[10] T. Tala et al., Density Peaking in JET: Driven by Fuelling or Transport?, (Institut de Recherche sur la Fusion par confinement Magntique (IRFM), Commissariat l2̌̃19nergie atomique (CEA/Cadarache), 13108 Saint-Paul-ls-Durance (France)) 26. IAEA Fusion Energy Conference. Programme, Abstracts and Conference Material

[11] P.T. Lang et al 2001 Nucl. Fusion 411107

[12] P T Lang et al 2002 Plasma Phys. Control. Fusion 441919

[13] L. R. Baylor et al., Physics of Plasmas 12, 056103 (2005); https://doi.org/10.1063/1.1865052

[14] B. Pegourie et al., (2018). Fueling DEMO: required flux and pellet injection parameters. Poster presented at 45th EPS Conference on Plasma Physics, Prague.

[15] C. Angioni et al 2007 Nucl. Fusion 471326

[16] M. Teschke et al., 2017 IEEE International Conference on Environment and Electrical Engineering and 2017 IEEE Industrial and Commercial Power Systems Europe (EEEIC / ICPS Europe), 6-9 June 2017, Milan (Italy) 
[17] Leuthold $\mathrm{N}$ et al 2018 On the relation between magnetic perturbations induced toroidal asymmetries and the pump-out effect in ASDEX Upgrade 45th EPS Conf. on Plasma Phys.

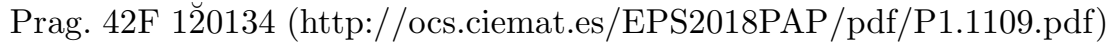

[18] R. Fischer et al., Fusion Sciente and Technology (58), 675 (2010)

[19] J. E. Rice et al., Physics of Plasmas 19, 056106 (2012)

[20] R.M. McDermott et al 2014 Nucl. Fusion 54043009

[21] I. Erofeev et al 2017 Nucl. Fusion 57126067

[22] Staebler G.M., Kinsey J.E. and Waltz R.E. 2005 Phys. Plasmas 12102508

[23] Staebler G.M., Candy J., Howard N.T. and Holland C. 2016 Phys. Plasmas 23062518

[24] G. V. Pereverzev et al., IPP Report 5/42 (August 1991)

[25] E. Fable et al., Plasma Phys. Control. Fusion 55, 124028 (2013)

[26] G.M. Staebler et al 2017 Nucl. Fusion 57066046

[27] G. Tardini et al 2007 Nucl. Fusion 47280

[28] J. Citrin, F. Jenko, P. Mantica, D. Told, C. Bourdelle, J. Garcia, J. W. Haverkort, G. M. D. Hogeweij, T. Johnson, and M. J. Pueschel Phys. Rev. Lett. 111, 15500120013 Published 7 October 2013; A. Di Siena et al 2018 Nucl. Fusion 58054002

[29] W. A. Houlberg et al., Phys. Plasmas (4), 3230 (1997)

[30] A. R. Polevoi, private communication (2001)

[31] R. Bilato et al 2011 Nucl. Fusion 51103034

[32] C. Angioni et al., Phys. Rev. Lett. (90), 205003 (2003)

[33] E Fable et al 2010 Plasma Phys. Control. Fusion 52015007

[34] T. Casper et al 2014 Nucl. Fusion 54013005

[35] Porcelli F, Boucher D and Rosenbluth M 1996 Plasma Phys. Control. Fusion 382163

[36] I T Chapman et al 2007 Plasma Phys. Control. Fusion 49 B385

[37] G.V. Pereverzev et al 2005 Nucl. Fusion 45221

[38] J.Y. Kim and H.S. Han, Phys. Plasmas 24, 072501 (2017)

[39] T. Hein et al., Physics of Plasmas 17, 102309 (2010); https://doi.org/10.1063/1.3503622

[40] A. Eriksson and J. Weiland, Physics of Plasmas 12, 092509 (2005)

[41] F. Sommer et al 2015 Nucl. Fusion 55033006

[42] E. Fable et al., Fus. Eng. and Design (130), 131 (2018) 